\title{
Creditworthiness and Thresholds in a Credit Market Model with Multiple Equilibria*
}

\author{
Lars Grüneł Willi Semmler ${ }^{\ddagger}$ and Malte Sieveking§ \\ Revised Version, October 2003
}

\begin{abstract}
The paper studies creditworthiness in a model with endogenous credit cost and debt constraints. Such a model can give rise to multiple candidates for steady state equilibria. We use new analytical techniques such as dynamic programming (DP) with flexible grid size to find solutions and to locate thresholds that separate different domains of attraction. More specifically, (1) we compute present value borrowing constraints and thus creditworthiness, (2) locate thresholds where the dynamics separate to different domains of attraction, (3) show jumps in the decision variable, (4) distinguish between optimal and non-optimal steady states, (5) demonstrate how creditworthiness and thresholds change with change of the credit cost function of the debtor and (6) explore the impact of debt ceilings and consumption paths on creditworthiness.
\end{abstract}

\footnotetext{
* An earlier version of this paper has been prepared for the 1998 North American Winter Meeting of the Econometric Society, January 1998, Chicago. We want to thank Jess Benhabib, Buz Brock, Gustav Feichtinger, Franz Wirl, Michael Woodford, Wolf-Jürgen Beyn and Thorsten Pampel for helpful discussions and comments on various versions of the paper. We also want to thank participants in a workshop at the University of Technology, Vienna, the Macroeconomic Workshop at Columbia University, and the SCE conference, at Yale University, June 2001. We are also grateful for comments from a referee of the journal.

${ }^{\dagger}$ Department of Mathematics, University of Bayreuth, Bayreuth, Germany, email:Lars.Gruene@uni-bayreuth.de

$\ddagger$ Center for Empirical Macroeconomics, Bielefeld and New School University, New York, NY 10003

$\S$ Department of Mathematics, University of Frankfurt, Frankfurt, Germany.
} 


\section{Introduction}

Numerous examples of dynamic economic models with multiple equilibria exist in the economic literature. ${ }^{1}$ Yet, only recently it has been discovered that the study of the local dynamics needs to be complemented by the study of the global dynamics. In those models there is history dependence in the sense that there are thresholds where the dynamics separate to different domains of attraction. Candidate of steady state equilibria may not be optimal and thresholds may or may not coincide with the candidates for steady state equilibria. Since there do not seem to exist equations to locate those thresholds, the thresholds need to be detected by applying numerical methods. We study here a simple economic model with borrower's and lender's relationship which gives rise to multiple candidates of steady state equilibria and thresholds. Although our model lends itself to a multi-variable interpretation to which our methods can be applied to, for analytical purpose we restrict our study to a one control-one state variable model.

In this simple variant capital stock is the state variable and investment is the control variable. We take into account temporary and intertemporal budget constraints of the agent who is allowed to finance investment through credit market borrowing. We allow for adjustment cost of capital and state dependent credit cost which can generate multiple candidates for steady state equilibria. Moreover, as in recent credit constraint models, we allow for debt ceilings. Our model resembles the dynamic models with credit market borrowing such as employed in Blanchard (1983), Bhandari et al. (1990), Kiyotaki and Moore (1997), Bernanke et al. (1999) and Miller and Stiglitz (1999). In these models the impact of credit market borrowing and debt dynamics on economic activity is studied. ${ }^{2}$

\footnotetext{
${ }^{1}$ Examples are given in the literature on development economics where a convex-concave production function arises which leads to a threshold that separates paths to low per capita income (poor) countries and high per capita income (rich) countries, see Skiba (1978) and Azariadis and Drazen (1990). In endogenous growth models of Lucas and Romer type multiple equilibria may arise by employing externalities or complementarities in the production function, for the Lucas model, see Benhabib, Perli and Xie (1994) and for the Romer model see Benhabib and Perli (1994) and Evans, Honkapohja and Romer (1997). On resources and the ecological management problem, see Brock and Starret (1999) and Sieveking and Semmler (1997); on trade theory, see Krugman (1991); on addiction, see Orphanides and Zervos (1998); on labor market search theory, see Mortensen (1989); and on monetary policy, see Benhabib, Schmitt-Grohe and Uribe (1998) and Greiner and Semmler (2002).

${ }^{2}$ In the above models, there is no study of thresholds. Note also that in contrast to
} 
Most of the literature on dynamic credit market models, by assuming perfect credit markets, posits that agents can borrow against future income as long as the discounted future income, the wealth of the agents, is no smaller than the debt that agents have incurred. There is no credit risk whenever the intertemporal budget constraint holds. Formally, often the so called transversality condition is invoked to provide a statement on the non-explosiveness of the debt of the economic agents. Models of this type have been discussed in the literature for households, firms, governments and countries (with access to international capital markets). ${ }^{3}$ Here then, as long as the intertemporal budget constraint holds, the intertemporal creditworthiness is automatically fulfilled for sufficiently small initial debt.

There are other studies that assume credit market imperfections so that borrowing is constrained. Borrowing ceilings are assumed which are supposed to prevent agents from borrowing an unlimited amount. Presuming that agents' assets serve as collateral a convenient way to define the debt ceiling is then to assume the debt ceiling to be a fraction of the agents' wealth. The definition of debt ceilings have become standard, for example, in small scale macro dynamic models. ${ }^{4}$

In other research, also building on imperfect capital markets it is posited that borrowers face a risk dependent interest rate which is assumed to be composed by a market interest rate (for example, risk-free interest rate) and an idiosyncratic component determined by the individual degree of risk of the borrower. Employing the theory of asymmetric information and costly state verification in Bernanke et al. (1999), for example, credit cost is endogenous by making it dependent on net worth of the borrower, as collateral for borrowing. ${ }^{5}$ This gives rise to an external finance premium that entrepreneurs have to pay contingent on their net worth. ${ }^{6}$

some of the other models, for example, the one by Bernanke et al.(1999) we, in order to simplify matters, do not employ a stochastic version but rather employ a deterministic framework. A stochastic version is discussed in Sieveking and Semmler (1999).

${ }^{3}$ For a brief survey of such models for households, firms and governments or countries, see Blanchard and Fischer (1989, ch.2) and Turnovsky (1995).

${ }^{4}$ See, for example Barro, Mankiw and Sala-i-Martin (1995). It has also been pointed out that banks (like the World Bank), often define debt ceilings for their borrowers, see Bhandari, Haque and Turnovsky (1990).

${ }^{5}$ Recent work has been undertaken by nesting credit market imperfections and endogenous borrowing cost more formally in intertemporal models such as the standard stochastic growth model, see Carlstrom and Fuerst (1997), Cooley and Quadrini (1998) and Krieger (1999). We restrict our study to a simple investment model.

${ }^{6}$ Another development of the analysis of credit risk employs less the "ability to pay" but rather the "willingness to pay" approach to explain defaults. For the latter type of literature, in particular on the problem of incentive compatible contracts, see Eaton and Fernandez (1995). 
We explore the global dynamics of a model when agents, as in the latter case, face endogenous credit cost. We also study the impact of debt constraints and debt ceilings on the global dynamics. To study global dynamics we have to compute creditworthiness. We show that debt ceilings should not be arbitrarily defined but rather given by creditworthiness. ${ }^{7}$ We use dynamic programming with flexible grid size to solve such type of models as well as to distinguish local from global dynamics. In particular, we (1) compute the present value borrowing constraint and creditworthiness without and with endogenous credit cost, (2) compute thresholds of those types of models where the dynamics separate to different domains of attraction, (3) show that the policy function may be discontinuous and compute the jumps in the policy function, (4) distinguish between optimal and non-optimal steady states and (5) demonstrate how the thresholds change with change of the credit cost function and (6) compute creditworthiness curves and thresholds for model variants with debt ceilings and given consumption paths.

We want to note that since in this paper we are concentrating on methodological issues such as history dependence, thresholds, domains of attraction, suboptimal equilibria and jumps in the policy function we use a stylized model which is nested in an intertemporal model with utility maximization but can be studied independently of utility maximization. The main property of our model is that the present value of the dynamic decision problem can be computed independently of the time path of consumption. For each time period dividend payments, or consumption, do not constrain the dynamic decision problem. There is, however, an intertemporal constraint for consumption. Yet the sequence of consumption decisions can be based on any preferences.

Finally we want to remark, that dynamic models with thresholds due to multiple steady state equilibria have been studied also in earlier work, such as Skiba (1978). ${ }^{8}$ Yet, most researchers have employed the Hamiltonian equation derived from Pontryagin's maximum principle. As shown in Beyn, Pampel and Semmler (2001) Pontryagin's maximum principle and the associated Hamiltonian can be applied to study the global dynamics of such a model with multiple steady states in restricted cases. In the current paper we propose the use of dynamic programming techniques on adaptively refined grids which is better suited to study a variety of model variants

The remainder of the paper is organized as follows. Section 2 introduces the basic dynamic model. Section 3 describes numerical methods, in par-

\footnotetext{
${ }^{7} \mathrm{~A}$ more elaborate analysis of how credit ceilings affects welfare is given in Semmler and Sieveking (1996).

${ }^{8}$ An extensive discussion of the earlier work on studying thresholds using the Hamiltonian is given in Brock and Malliaris (1996, ch. 6)
} 
ticular dynamic programming with flexible grid size that are used to study different variants of the dynamic model. Sections 4 reports the detailed results from our numerical study on the different variants of the model. Section 5 concludes the paper. $^{9}$

\section{The Dynamic Model}

Next we want to specify the dynamic model that we study analytically and numerically. As above mentioned in the study of creditworthiness we can by-pass utility theory though the model can be nested in a model with utility theory. Economists have argued that analytical results in intertemporal models frequently depend on the form of the utility function employed. We show that we can study borrowing, lending and creditworthiness, without the direct use of utility theory. Although our model can be nested in utility theory, we use a separation theorem that permits us to separate the present value problem from the consumption problem. ${ }^{10}$

Note that by focusing on a dynamic decision problem, an optimal investment problem, where debt can be continuously issued and retired we do not have to consider that in each period the agent is constrained by financial constraints but there will be intertemporal debt constraints where debt capacity will be defined by the agent's creditworthiness and credit constraints. Moreover, due the aforementioned separation theorem, dividend payment, or consumption, represent no constraint on the dynamic decision problem. Substracting initial debt obligation, any present value generated by the sequence of investment decisions can finally be consumed. The sequence of consumption decisions faces an intertemporal constraint, but the consumption path can be determined by any preference.

As to the more specifics of the credit market features of our model we presume credit market imperfections. Along the line of Bernanke, Gertler and Gilchrist (1999), henceforth BGG, we assume asymmetric information and agency costs in borrowing and lending relationships. BGG draw on the insight of the literature on costly state verification ${ }^{11}$ in which lenders must pay a cost in order to observe the borrower's realized returns. This motivates the use of collaterals in credit market models. Uncollateralized borrowing is

\footnotetext{
${ }^{9}$ For the more extensive version of the generalized model, see Semmler and Sieveking (1999).

${ }^{10}$ In Sieveking and Semmler (1998) a more analytical treatment is given of why and under what conditions the subsequent credit market model can be separated from the consumption problem.

${ }^{11}$ This literature originates in the seminal work by Townsend (1979).
} 
assumed to pay a larger finance premium than collateralized borrowing or self-financing. The external finance premium is interpretable as the cost of bankruptcy (for example constituted by auditing, accounting, legal cost, as well as loss of assets arising from asset liquidation). Thus the external finance premium frequently also called default premium, drives a wedge between the expected return of the borrower and the risk-free interest rate whereby the premium is positively related to the default cost and inversely related to the borrowers net worth. Net worth is defined as the agent's collateral value of the (illiquid) capital stock less the agent's outstanding obligations.

Following BGG we can measure the inverse relationship between the external finance premium and net worth in a function such as

$$
H(k, B)=\frac{\alpha_{1}}{\left(\alpha_{2}+\frac{N}{k}\right)^{\mu}} \theta B
$$

with $H(k, B)$ the finance premium depending on net worth, $N=k-B$, with $k$ ( as capital stock and $B$ as debt. For the subsequent use we may write $\frac{N}{k}=1-\frac{B}{k}$. The parameters are $\alpha_{1}, \alpha_{2}, \mu>0$ and $\theta$ is the risk-free interest rate. Note that this function implicitly constrains the state $(k, B)$ by $B<\left(\alpha_{2}+1\right) k$, since $H(k, B)$ tends to infinity as $B$ tends to $\left(\alpha_{2}+1\right) k$. In the analytical and numerical study of the model below we presume that the external finance premium will be zero for $N=k$ and thus for $B(t)=0^{12}$ so that in the limit the borrowing rate is the risk-free rate.

Alternatively, we will also consider a credit cost that is simply convex in the level of debt. Note, however, that even if the credit cost is endogenized we might want to define the agent's financial constraints which in our model will be given by an upper bound of the debt-capital ratio, see section 4 .

Employing the above theory of endogenous credit cost based on net worth, we study the following intertemporal model with credit market borrowing

$$
\begin{gathered}
V(k)=\operatorname{Max}_{j} \int_{0}^{\infty} e^{-\theta t} f(k(t), j(t)) d t \\
\dot{k}(t)=j(t)-\sigma k(t), \quad k(0)=k . \\
\dot{B}(t)=H(k(t), B(t))-(f(k(t), j(t))-c(t)), B(0)=B_{0}
\end{gathered}
$$

\footnotetext{
${ }^{12}$ Bernanke, Gertler and Gilchrist (1999) employ the same functional relationship as above. They state that ".. the external finance premium depends inversely on the share of the firm's capital investment that is financed by the entrepreneur's own net worth" (p.166).
} 
The model represents an optimal investment problem with adjustment cost of capital and endogenous credit cost. In addition, debt constraints can be imposed. The agent's net income

$$
f(k, j)=a k^{\alpha}-j-j^{\beta} k^{-\gamma}
$$

is generated from capital stock, through a production function, $a k^{\alpha}$, and investment, $j$, is undertaken so as to maximize the present value of net income in (5) given the adjustment cost of capital $j^{\beta} k^{-\gamma}$. Note that $\sigma>0, \alpha>0, \beta>$ $1, \gamma>0$, are constants. ${ }^{13}$ Equ. (3) represents capital accumulation and equ. (4) the evolution of debt of the economic agent. We allow for investment rates $j \geq 0$. If we take $\beta=2$ and $\gamma=0$ we have the standard model with quadratic adjustment cost of investment. Instead of $a k^{\alpha}$ we may also employ a convex-concave production function and drop the adjustment cost term $j^{\beta} k^{-\gamma}$ as can be found in Skiba (1978).

Note that in (4) $c(t)$ is a consumption stream that is, in the context of our model, treated as exogenous. ${ }^{14}$ Below the consumption stream will be specified further. Since net income in (5) less the consumption stream $c(t)$ can be negative the temporary budget constraint of the agent requires further borrowing from credit markets and if there is positive net income less consumption debt can be retired.

In equ. (4), along the line of the theory of imperfect capital markets, we assume that the credit cost $H(k, B)$ may be state dependent, depending on the capital stock, $k$, and the level of debt $B$ with $H_{k}>0$ and $H_{B}<0$. Note, however, that if we assume that credit cost depends inversely on net worth ${ }^{15}$ as, for example, in equ. (1) we get a special case of our model when only the risk-free interest rate is accounted for in the credit cost.

To begin with the simplest case we take a constant interest rate. The optimal investment problem reads

\footnotetext{
${ }^{13}$ Note that the production function $a k^{\alpha}$ has a scaling factor. For the analytics we leave it aside. Other authors have used the simplification $H(k, B)=\theta B, \beta=2, \gamma=1$ to study such a model, see Blanchard (1983).

${ }^{14}$ Note that in our model all variables are written in efficiency labor, therefore $\sigma$ represents sum of the capital depreciation rate, population growth and rate of exogenous technical change. Our model resembles the one by Blanchard (1983) but builds on imperfect capital markets and thus it endogenizes credit cost.

${ }^{15}$ In computing the present value of the future net income we do not have to assume a particular fixed interest rate, as in Kiyotaki and Moore (1997) and Miller and Stiglitz (1999).
} 


$$
\begin{array}{r}
\max _{j} \int_{0}^{\infty} e^{-\theta t}(f(k(t), j(t)) d t \\
\dot{k}=j-\sigma k \quad k(0)=k \\
j \geq 0 \\
\dot{B}=\theta B-f(k, j)
\end{array}
$$

The above model holds regardless of $B(t)$. We call the maximized integral the present value of $k$.

One then may ask the following two questions. (1) why is $V(k)<\infty$ ? and $(2)$ is $\sup B(t)<\infty$ ?

The answer is given by partial integration of (6) which is

$$
\int_{0}^{T} e^{-\theta t} f(k, j) d t=B(0)-e^{-\theta T} B(t) .
$$

If $j$ solves the optimal investment problem, then we obtain

$$
V(k)=B(0)-\lim _{T \rightarrow \infty} e^{-\theta T} B(T)
$$

Hence, if $V(k)-B(0)>0$ then $B(T)<0$ eventually, but if $V(k)-B(0)<$ 0 then $\lim _{T \rightarrow \infty} e^{-\theta T} B(T)>0$, and $B(t)$ increases exponentially.

Hence, in solving the optimal investment problem the intertemporal budget constraint is automatically satisfied provided that

$$
B(0)<V(k)
$$

but $B(t)$ increases exponentially, if $B(0)>V(k)$, no matter how $j$ is chosen. Therefore we might define creditworthiness of $k$ by $B^{*}(k)=V(k)$ and incorporate the state constraint $B(0)<B^{*}(k)$ as well as (6) into the optimal investment problem.

Next let us consider consumption. Suppose the problem is to optimize welfare

$$
\begin{gathered}
\max _{c, j} \int_{0}^{\infty} e^{-\theta_{1} t} U(c(t)) d t \\
\dot{k}=j-\sigma k \\
k(0)=k \\
\dot{B}=\theta B-f(k, j)+c, \quad B(0)=B
\end{gathered}
$$


Note that in the latter case, we have two control variables $j, c$. Partial integration of the second differential equation this time gives

$$
\max _{c, j} \int_{0}^{T} e^{-\theta t} f(k, j) d t=B(0)-B(T) e^{-\theta T}+\int_{0}^{T} e^{-\theta t} c(t) d t
$$

Hence, if $V(k)-B(0)>0$

$$
\begin{aligned}
& V(k)=B(0)-\lim e^{-\theta T} B(T)+\int_{0}^{\infty} e^{-\theta t} c(t) d t \\
& V(k)=B(0)+\int_{0}^{\infty} e^{-\theta t} c(t) .
\end{aligned}
$$

The above suggests a two stage procedure: (1) solve the optimal investment problem provided that $V(k)>B(0)$ and (2) solve

$$
\begin{gathered}
\max _{c} \int_{0}^{\infty} e^{-\theta_{1} t} U(c(t)) \\
\text { s.t. } \quad \int_{0}^{\infty} e^{-\theta t} c(t) d t=V(k)-B(0) .
\end{gathered}
$$

Now suppose ${ }^{16}$ instead of $\theta B$ we have an external finance premium $H(k, B)$ as in equ. (1), and presume first

$$
\dot{B}=H(k, B)-f(k, j) \quad(c=0)
$$

Definition: Call the pair of initial capital and debt $(k, B)$ creditworthy if there is an investment rate $j(t)$ such that the solution to

$$
\begin{aligned}
\dot{k} & =j-\sigma k \\
\dot{B}(t) & =H(k, B)-f(k, j), \quad B(0)=B .
\end{aligned}
$$

satisfies $B(t) \leq 0$ for some $t>0 . B^{*}(k)=\sup \{B \mid(k, B)$ creditworthy $\}$ is called creditworthiness of $k$. Note that this definition of creditworthiness excludes the points on the critical curve $B^{*}(k)$ itself, since, as it turns out, it is impossible to drive a state $\left(k(0), B^{*}(k(0))\right)$ with $B^{*}(k(0))>0$ to $(k(t), 0)$ in finite time $t$.

\footnotetext{
${ }^{16}$ Note that the constraint expressed in equ. (8) is, in the case $B(0)=0$, represents the intertemporal consumption based asset pricing model, whereby in equ. (8) the discount rate, $\theta$, is a constant, and not derived from the growth rate of marginal utility of consumption. How the equ. (8) looks like presuming the latter discount rate, see Grüne and Semmler (2003).
} 
Next, we add the intertemporal budget constraint $B \leq B^{*}(k)$ to the optimal investment problem. The form of $H(k, B)$ represents our attempt to capture real credit market features whereby the above inclusion of an upper borrowing limit, $B<k\left(\alpha_{2}+1\right)$, excludes debt explosiveness.

Our next task is to analyze creditworthiness and its relation to the present value as well as feasible consumption. We use the more general formulation of credit cost as given in equ. (1) but it can include the case $H(k, B)=\theta B$. We define extremal investment rates $j_{ \pm}$to decrease $B$ as much as possible while in(de)creasing capital:

Definition: Suppose $H(k, B)>f(k, \sigma k)$ then $j_{+}(k, B)$ is defined by

$$
\underset{j>\sigma k}{\operatorname{Min}} \frac{H(k, B)-f(k, j)}{j-\sigma k}=\frac{H(k, B)-f\left(k, j_{+}(k, B)\right)}{j_{+}(k, B)-\sigma k}
$$

and, respectively, $j_{-}(k, B)$ is defined by

$$
\underset{j<\sigma k}{\operatorname{Max}} \frac{H(k, B)-f(k, j)}{j-\sigma k}=\frac{H(k, B)-f\left(k, j_{-}(k, B)\right)}{j_{-}(k, B)-\sigma k}
$$

We want to remark that we use $\beta>1$ to prove that $j_{ \pm}$are uniquely defined and locally Lipschitz. Suppose that $H(k, B)>f(k, \sigma k)$, then there is a unique solution

$$
\left(x_{ \pm}(t, k, B), y_{ \pm}(t, k, B)\right)=(x(t), y(t))
$$

to

$$
\dot{x}=j_{ \pm}-\sigma x, \quad \dot{y}=H(x, y)-f\left(x, j_{ \pm}(x, y)\right), \quad x(0)=k, \quad y(0)=B .
$$

Note that $x_{+}(t, k, B)$ as well as $y_{+}(t, k, B)$ are increasing functions of $t$. Hence, the following definition makes sense.

\section{Definition:}

$$
B^{+}(k)=\sup \left\{B \mid \lim _{t} y_{+}(t, k, B)<\infty\right\}
$$

Note that $x_{-}(t, k, B)$ is decreasing while $y_{-}(t, k, B)$ is decreasing or first decreasing and then increasing. Therefore we may define

$$
B^{-}(k)=\sup \left\{B \mid \lim _{t} y_{-}(t, k, B)<\infty\right\}
$$


We have the following theorem as should be intuitive clear to the reader from a sketch of the set $H(k, B) \leq \sigma k$, see figure 1 .

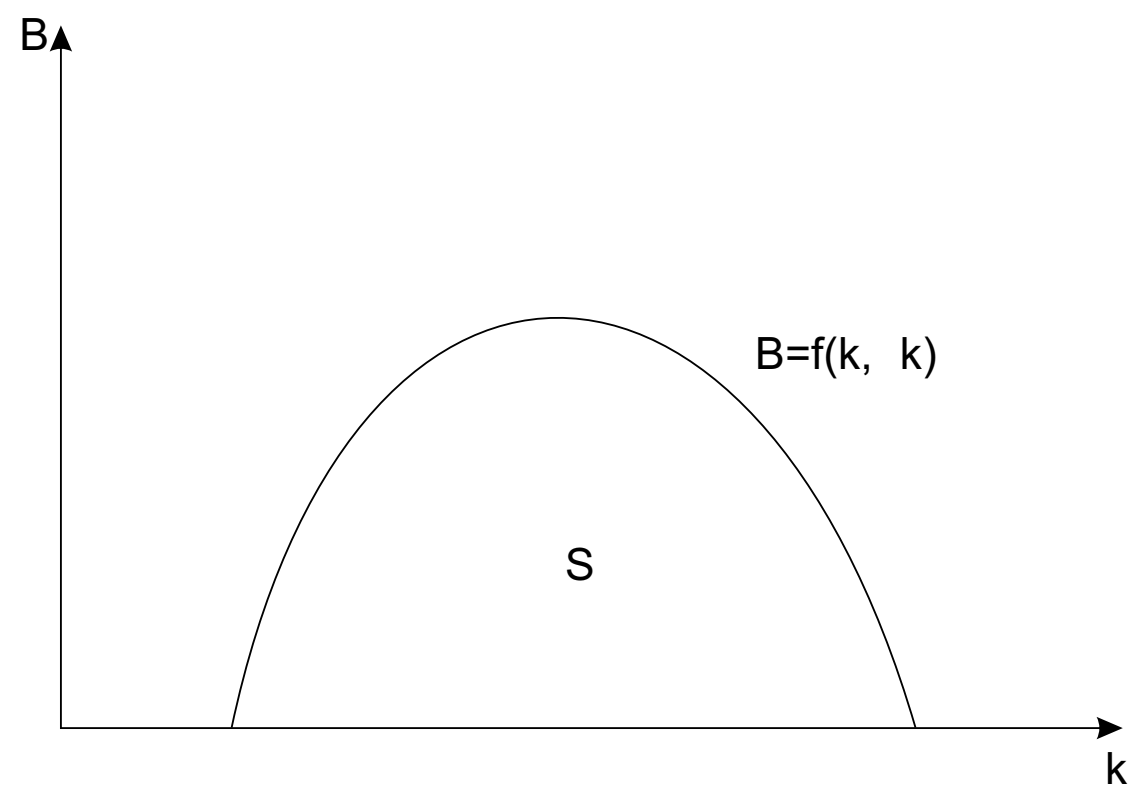

Figure 1: The $\theta B=f(k, \sigma k)$ curve

\section{Theorem:}

$$
B^{*}(k)=\max \left(B^{+}(k), B^{-}(k)\right)
$$

Corollary 1: Creditworthiness $\left\{\left(k, B^{*}(k)\right) \| k \geq 0\right\}$ is composed of a solution to the optimal investment problem with initial values $\left(k(0), B^{*}(k(0))\right)=$ $(k(0), B(0))$.

Stationary states $\left(k, B^{*}(k)\right.$ have to satisfy $H(k, B)=f(k, \sigma k)$. In case $H(k, B)=\theta B$ the boundary of the set $S=\{(k, B) \mid H(k, B)<f(k, \sigma k)\}$ is the graph of a function such as represented in figure 1 .

Moreover, if creditworthiness $\left(k, B^{*}(k)\right)$ touches $S$ we have the following equation:

$$
\begin{aligned}
\frac{H(k, B)-f\left(k, j_{+}\right)}{j_{+}-\sigma k} & =-\frac{\partial f}{\partial j}\left(k, j_{+}\right)=1+\beta \sigma^{\beta-1} k^{\beta-\gamma-1} \\
& =\frac{\partial}{\sigma k}\left(\theta^{-1} f(k, \sigma k)\right) \text { in case } H(k, B)=\theta B .
\end{aligned}
$$

In general if $G=H(k, B)-f(k, \sigma k)$ 


$$
\frac{\partial G}{\partial k}+\frac{\partial G}{\partial B} \frac{\partial f}{\partial j}=0, \quad G=0
$$

Corollary 2: Steady states $\left(k, B^{*}(k)\right)$ satisfy

$$
\frac{\partial G}{\partial k}+\frac{\partial G}{\partial B} \frac{\partial f}{\partial j}=0, \quad G=0
$$

which for $H(k, B)=\theta B$ gives

$$
\frac{d}{d k} f(k, \sigma k)+\theta \frac{\partial f}{\partial j}(k, \sigma k)=0
$$

Next we explore how creditworthiness $B^{*}(k)$ relates to the present value of $k$ - and consumption - now that we imposed $B(t) \leq B^{*}(k(t))$ defined by a finance premium $H(k, B)>\theta B$. The answer is again obtained by considering the equation

$$
\dot{B}=H(k, B)-f(k, j) .
$$

Multiplying by $e^{-\theta t}$ and using partial integration we find

$$
\int_{0}^{T} e^{-\theta t} f(k, j) d t=B(0)-e^{-\theta T} B(T)+\int_{0}^{T} e^{-\theta t}(H-\theta B) d t
$$

Let us define the present value of the external finance premium - with initial value $(k, B)$ - by

$$
\int_{0}^{\infty} e^{-\theta t}(H-\theta B) d t=V_{H}(k, B) .
$$

where we use the optimal investment rate $j$. Then for $T \rightarrow \infty$ we find

$$
V(k)=B(0)-\lim e^{-\theta T} B(T)+V_{H}(k, B)
$$

Note that we permit $B(t)$ to be negative. The term $V_{H}(k, B)$ would be equal to zero for the case $H(k, B)=\theta B .{ }^{17}$

In particular, if $B(0)=B^{*}(k)$ we have

$$
V(k)=B^{*}(k)+V_{H}\left(k, B^{*}(k)\right)
$$

\footnotetext{
${ }^{17}$ Note that in practise the discount rate $\theta$ is often approximated by taking a weighted average cost of capital (WACC), composed of the weighted average of equity return and bond return, see Benninga (2000, ch. 2), see also Abel and Blanchard (1986).
} 
The quantity lim $e^{-\theta T} B(T)$ measures feasible consumption. Considering now consumption we can write

$$
\begin{aligned}
\dot{B} & =H(k, B)-f(k, j)+c \text { and } \\
\int_{0}^{T} e^{-\theta t} f(k, j) d t & =B(0)-e^{-\theta T} B(T)+\int_{0}^{T} e^{-\theta t} c d t+\int_{0}^{t} e^{-\theta t}(H-\theta B) d t
\end{aligned}
$$

Suppose $c(t)$ is chosen in such a way that

$$
\lim e^{-\theta T} B(T)=0
$$

This is feasible. Then,

$$
V(k)=B(0)+\int_{0}^{\infty} e^{-\theta t} c(t) d t+V_{H}(k, B)
$$

Hence, $c(t)$ is feasible if and only if

$$
\int_{0}^{\infty} e^{-\theta t} c(t) d t \leq V(k)-B(0)-V_{H}(k, B)
$$

and we see that $V_{H}(k, B)$ can be interpreted as welfare loss that constitutes the cost for the risk-insurance arising from default risk. ${ }^{18}$

The preceding characterization of feasible consumption may again be stated as a separation theorem: the optimal consumption may be computed by a two stage optimization - first, compute the optimal investment and thereby $V(k)-B(0)-V_{H}(k, B)$, then, second, compute $\sup \{U(c) \mid c$ feasible\}. ${ }^{19}$

Finally note, that in the case of endogenous credit cost the Modigliani and Miller theorem does not hold. Thus, borrowing with a finance premium affects the value of the firm. This is made clear in the following remark 1 , see also sect. 4.3 .

Remark 1: We want to point out how to compute $V(k)$ and its component parts. We can use the above theorem to compute the solution to the optimal investment problem:

\footnotetext{
${ }^{18}$ Note that in the latter integral, asset prices and consumption are not determined as in equ. (8) but the finance premium $V_{H}(k, B)$ has to be taken into account.

${ }^{19} \mathrm{We}$ want to note here that, strictly speaking, this statement only holds if $B$ represents outside debt(liabilities to outside creditors). For inside debt, of course, the consumption stream within a country can also be financed through investment in bonds, $B$, generating an income and thus consumption stream for a creditor. In the latter case what is consumable then is $V(k)=B^{*}(k)$.
} 
1. Compute $B^{*}(k)$, thereby find the optimal path $(k(t), B(t))$ which satisfies $B(t)=B^{*}(k(t))$.

2. Compute

$$
V_{H}(k, B)=\int_{0}^{\infty} e^{-\theta t}(H(k(t), B(t))-\theta B(t)) d t
$$

3. Compute

$$
V(k)=B^{*}(k)+V_{H}\left(k, B^{*}(k)\right) .
$$

Remark 2: The particular form of our production function is not decisive for our results, nor the particular form of $H(k, B)$. What is decisive is that the optimal value function is finite.

Remark 3: Note that we are considering here a deterministic case where the present value is achievable if $B(0)<B^{*}(k)$ and which is impossible to achieve for $B(0)>B^{*}(k)$. In case there are stochastic influences the realization of the present value will always be impossible to achieve with a positive probability and hence the $B(t)$ will influence the optimal investment $j(t)$ in all times.

In sum, Remark 1 allows to compute the creditworthiness, for the case $H(k, B)=\theta B$ where $V(k)=B^{*}(k)$ and for the case of an external finance premium $H(k, B)$, where for $H\left(k, B^{*}(k)\right)$ the creditworthiness will be less than $V(k)$. We also can consider the case when there are fixed debt ceilings.

\section{$3 \quad$ Numerical Dynamic Programming}

In the literature it has been shown that dynamic optimization models giving rise to multiple steady state equilibria can be of concave ${ }^{20}$ or non-concave type and yet generating multiple equilibria with thresholds. ${ }^{21}$ Although most of the historical models, as discussed in the introduction, build on non-concave models, yet recently examples have been given where such phenomena can also arise in concave models. ${ }^{22}$ In the case of the existence

\footnotetext{
${ }^{20}$ The problem is concave if, following the Mangasarian definition, the Hamiltonian for the above problem is both concave in the state as well as control variables.

${ }^{21}$ In the development literature such a threshold - which has, however, been identified with the middle unstable steady state - has been called a development trap, see for example, Azariadis and Drazen (1990).

${ }^{22}$ See, for example, Hartl et al. (2003), and Deissenberg et al. (2003).
} 
of multiple steady state equilibria of system (2)-(4) a rigorous study of the dynamics of the model and the thresholds were the dynamics separate to different domains of attraction would require locating the thresholds analytically. This appears to be feasible only if the thresholds coincide with one of the steady state equilibria. As has been shown this occurs if the relevant (unstable) equilibria is a node. In the concave model the unstable equilibria is necessarily a node, but a node can also occur in a non-concave model. ${ }^{23}$ Yet, it is impossible to locate the threshold analytically if the threshold does not coincide with the (unstable) equilibria. Thus, the thresholds that will exists in the vicinity of the unstable steady state - and will render the neighboring unstable steady state to be non-optimal- have to be located by numerical methods. ${ }^{24}$

In this section we describe two dynamic programming algorithms which enable us to compute the creditworthiness curve $B^{*}(k)$ and the thresholds. While the two algorithms presented here are of quite different nature, a common feature of both is the adaptive discretization of the state space which leads to high numerical accuracy with moderate use of memory.

\subsection{The Discounted Infinite Horizon Problem}

The first algorithm is applied to discounted infinite horizon optimal control problems of type (2)-(4) when, however, no restriction on the dynamics is present. In our model, this applies if the credit cost is constant, i.e., $H(k, B)=\theta B$ as in (6) and if in addition no explicit constraint on $B(t)$ is imposed, since in this case it follows that $B^{*}(k)$ is easily obtained from $V(k)$ in (2), namely

$$
B^{*}(k)=V(k)=\operatorname{Max}_{j} \int_{0}^{\infty} e^{-\theta t} f(k(t), j(t)) d t
$$

cf. the discussion after (6).

We will briefly describe the algorithm which goes back to Capuzzo Dolcetta (1983), Falcone (1987) and Grüne (1997). For details and for a mathematically rigorous convergence analysis we refer to these papers as well as to Appendix A in the monograph by Bardi and Capuzzo Dolcetta (1997) and to Grüne, Metscher and Ohlberger (1999).

\footnotetext{
${ }^{23}$ See Hartl et al. (2003) and Deissenberg et al. (2003).

${ }^{24}$ Note that for a constant interest rate we might apply the Hamiltonian from Pontryagin's maximum principle to determine the optimal investment. Yet, if there is more than one positive candidate for an optimal stationary capital stock by local analysis, one would get not information that one of those (and which) is non-optimal.
} 
In the first step, the continuous time optimal control problem is replaced by a first order discrete time approximation given by

$$
V_{h}(k)=\operatorname{Max}_{j} J_{h}(k, j), \quad J_{h}(k, j)=h \sum_{i=0}^{\infty}(1-\theta h)^{i} f\left(k_{h}(i), j_{i}\right)
$$

where $k_{h}$ is defined by the discrete dynamics

$$
k_{h}(0)=k, \quad k_{h}(i+1)=k_{h}(i)+h\left(j_{i}-\sigma k_{h}(i)\right)
$$

and $h>0$ is the discretization time step. Note that $j=\left(j_{i}\right)_{i \in \mathbb{N}_{0}}$ here denotes a discrete control sequence.

The optimal value function is the unique solution of the discrete HamiltonJacobi-Bellman equation

$$
V_{h}(k)=\operatorname{Max}_{j}\left\{h f\left(k, j_{0}\right)+(1-\theta h) V_{h}\left(k_{h}(1)\right)\right\},
$$

where $k_{h}(1)$ denotes the discrete solution corresponding to the control $j$ and initial value $k$ after one time step $h$. Abbreviating

$$
T_{h}\left(V_{h}\right)(k)=\operatorname{Max}_{j}\left\{h f\left(k, j_{0}\right)+(1-\theta h) V_{h}\left(k_{h}(1)\right)\right\}
$$

the second step of the algorithm now approximates the solution on a grid $\Gamma$ covering a compact subset of the state space, i.e., a compact interval $[0, K]$ in our setup. Denoting the nodes of $\Gamma$ by $k^{i}, i=1, \ldots, P$, we are now looking for an approximation $V_{h}^{\Gamma}$ satisfying

$$
V_{h}^{\Gamma}\left(k^{i}\right)=T_{h}\left(V_{h}^{\Gamma}\right)\left(k^{i}\right)
$$

for each node $k^{i}$ of the grid, where the value of $V_{h}^{\Gamma}$ for points $k$ which are not grid points (these are needed for the evaluation of $T_{h}$ ) is determined by linear interpolation. We refer to the papers cited above for the description of iterative methods for the solution of (16). Note that an approximately optimal control law (in feedback form for the discrete dynamics) can be obtained from this approximation by taking the value $j^{*}(k)=j$ for $j$ realizing the maximum in (14), where $V_{h}$ is replaced by $V_{h}^{\Gamma}$. This procedure in particular allows the numerical computation of approximately optimal trajectories.

In order to distribute the nodes of the grid efficiently, we make use of a posteriori error estimation. For each cell $C_{l}$ of the grid $\Gamma$ we compute

$$
\eta_{l}:=\underset{k \in C_{l}}{\operatorname{Max}}\left|T_{h}\left(V_{h}^{\Gamma}\right)(k)-V_{h}^{\Gamma}(k)\right|
$$

(more precisely we approximate this value by evaluating the right hand side in a number of test points). It can be shown that the error estimators $\eta_{l}$ 
give upper and lower bounds for the real error (i.e., the difference between $V_{h}$ and $V_{h}^{\Gamma}$ ) and hence serve as an indicator for a possible local refinement of the grid $\Gamma$. It should be noted that this adaptive refinement of the grid is very effective ${ }^{25}$ for detecting thresholds, because the optimal value function typically fails to be differentiable in these points, resulting in large local errors and consequently in a fine grid, see Figure 5.

\subsection{Domains of Attraction}

For the general model, i.e., with endogenous credit cost $H(k, B)$ as defined in (1) and/or restrictions of the type $B / k \leq c$, this algorithm unfortunately is not applicable. Even though in certain cases a HJB equation for a discrete time version of the problem is available, it is not clear whether the full discretization procedure described above leads to a valid and convergent approximation of $B^{*}$

Hence we propose a different approach for the solution of this problem, based on a set oriented method for the computation of domains of attraction. The method relies on the following observation: define the set

$$
D=\left\{\begin{array}{l|l}
\left(k_{0}, B_{0}\right) & \begin{array}{l}
\text { there exists } j \text { such that } B(t(j)) \leq 0 \\
\text { for some } t(j)>0
\end{array}
\end{array}\right\} .
$$

Then the curve $B^{*}(k)$ is exactly the upper boundary of $D$. Note that explicit debt ceilings of the type $B(t) \leq c k(t)$ are easily incorporated into this setup by considering only those trajectories in the definition of $D$ which meet this restriction.

The set $D$ is what is called a controlled domain of attraction of the set $A=\left\{(k, B) \in \mathbb{R}^{2} \mid B \leq 0\right\}$ and we will now give a brief description of an algorithm for the computation of such sets, for details we refer to Grüne (2001) and Grüne (2002, ch. 7).

Again we consider a first order discrete time approximate model, now both for $k$ and $B$ given by the Euler discretization ${ }^{26}$

$$
\begin{aligned}
k_{h}(i+1) & =k_{h}(i)+h\left(j_{i}-\sigma k_{h}(i)\right) \\
B_{h}(i+1) & =B_{h}(i)+h H\left(k_{h}(i), B_{h}(i)\right)-h f\left(k_{h}(i), j_{i}\right)
\end{aligned}
$$

\footnotetext{
${ }^{25}$ Actually, for the one-dimensional problem at hand it is possible to compute rather accurate approximations $v_{h}^{\Gamma}$ also with equidistributed grid points. In higher dimensions the computational advantage of adaptive gridding is much more obvious, see, e.g., the examples in Grüne (1997) or Grüne et al. (1999).

${ }^{26}$ We use the simple first order Euler scheme here in order to avoid too much technicality in our presentation. For higher order discrete time approximations see, e.g., Grüne (2002, ch. 5).
} 
and abbreviate the right hand side by $\Psi(k, B, j)$. Just as above, for the space discretization we use a grid $\Gamma$, now covering a two-dimensional rectangular domain $[0, K] \times[0, \bar{B}]$. For each cell $C_{l}, l=1, \ldots, Q$ of the grid we use a collection of test points $x_{l}^{i}=\left(k_{l}^{i}, B_{l}^{i}\right), i=1, \ldots, N$ in order to compute the set image

$$
\Phi\left(C_{l}, \bar{\jmath}\right)=\bigcup_{m} C_{m} \text { for all } m \in\{1, \ldots, Q\} \text { with } \Psi\left(k_{l}^{i}, B_{l}^{i}, j^{i}\right) \in C_{m}
$$

where $\bar{\jmath}=\left(j^{1}, \ldots, j^{N}\right)$ is a vector of $N$ control values associated to the $N$ test points. For a sequence $\left(\bar{\jmath}_{i}\right), i=0,1,2, \ldots$ of such control vectors we can iterate the map $\Phi$ and we denote the resulting iterated map by $\Phi_{i}\left(C_{l},\left(\bar{\jmath}_{i}\right)\right)$. Now we can define the following three sets

$$
\begin{aligned}
& D_{\Gamma}=\bigcup_{m} C_{m} \text { for all } m \text { with } \Phi_{i}\left(C_{m},\left(\bar{\jmath}_{i}\right)\right) \subseteq A \text { for some }\left(\bar{\jmath}_{i}\right) \text { and some } i \\
& B_{\Gamma}=\bigcup_{m} C_{m} \text { for all } m \text { with } \Phi_{i}\left(C_{m},\left(\bar{\jmath}_{i}\right)\right) \cap A=\emptyset \text { for all }\left(\bar{\jmath}_{i}\right) \text { and all } i \\
& E_{\Gamma}=\bigcup_{m} C_{m} \text { for all } m \text { with } C_{m} \nsubseteq D_{\Gamma} \text { and } C_{m} \not E_{\Gamma}
\end{aligned}
$$

These sets are easily computed by a dynamic programming type iteration and under appropriate conditions it can be shown that the set $D_{\Gamma}$ approximates $D$, the set $B_{\Gamma}$ approximates $D^{c}$ (the complement of $D$ ) and the set $E_{\Gamma}$ approximates $\partial D$ (the boundary of $D$ ), which in our case is exactly the curve $B^{*}(k)$. It turns out that for obtaining more and more accurate approximations (with respect to the space discretizations) it is sufficient to increase the accuracy on the set $E_{\Gamma}$, i.e., to refine the cells $C_{m} \subseteq E_{\Gamma}$.

While the convergence analysis in the general case is rather complicated and depends on certain properties of $D$, for our problem we can use the fact that the boundary $\partial D$ is given by the curve $B^{*}(k)$ which is monotone increasing in $k$. Hence, if we use a rectangular grid, and choose the test points in each cell to be the 4 corners of this rectangular cell, we obtain that if a cell $C_{m}$ intersects both $D$ and $D^{c}$, then there exist test points $x_{m}^{k_{1}}$ and $x_{m}^{k_{2}}$ in this set such that $x_{m}^{k_{1}} \in D$ and $x_{m}^{k_{2}} \notin D$. Consequently, the iterated cell image $\Phi_{i}$ cannot be contained in $A$ for any $\left(\bar{\jmath}_{i}\right)$ (implying that $C_{m} \nsubseteq D_{\Gamma}$ ) but it intersects $A$ for some $\left(\bar{\jmath}_{i}\right)$ (implying that $C_{m} \nsubseteq B_{\Gamma}$ ). Thus, if a cell $C_{m}$ intersects both $D$ and $D^{c}$ then we obtain that $C_{m} \subseteq E_{\Gamma}$ which finally yields that the set $E_{\Gamma}$ always covers the boundary $\partial D$ and hence gives an approximation of the curve $B^{*}(k)$ whose accuracy is equal to the width of the set $E_{\Gamma} \cdot{ }^{27}$

\footnotetext{
${ }^{27}$ Of course, this discussion concerns the spatial discretization error only. For the analysis of the full error we refer to the cited references.
} 
Since the problem which is solved by this algorithm is not a classical optimal control problem (though it can be interpreted as an optimal control problem for the set valued dynamics) it is not possible to obtain optimal trajectories with respect to some given functional. However, it is not too difficult to prove that the boundary of a domain of attraction $D$ is weakly invariant (i.e., for an initial value on the boundary $\partial D$ we can always find trajectories that remain on $\partial D$ for all future times), provided it is a "proper" domain of attraction, i.e., its boundary does not intersect with the boundary of $A$ and provided that no additional restrictions apply. Due to this fact, for each initial value $(k, B) \in D_{\Gamma}$ (recall that this set forms our numerical approximation of the set $\left\{(k, B) \mid B<B^{*}(k)\right\}$ of subcritical initial values) we can compute a control sequence $j_{i}$ realizing a (discrete time) trajectory for which $B_{h}(i)$ remains bounded for all times $i \geq 0$ and for initial values on the upper part of the boundary $\partial D_{\Gamma}$ we can even expect to find trajectories that stay on the boundary $\partial D_{\Gamma}$ for all future times, i.e., they are (up to the numerical error) of the form $\left(k(t), B^{*}(k(t))\right.$. The limiting behavior of these trajectories can then be used for the detection of the thresholds and it turns out that this procedure yields very good results.

\section{The Numerical Study}

In this section we present numerical results for different variants of our model (2)-(4). The differences pertain to the production function, adjustment cost of capital and the choice of $H(k, B)$. Throughout this section we specify the model parameter $\sigma=0.15$ and $\gamma=0.3$. Other parameters will be model specific. Unless otherwise noted we first use $c(t) \equiv 0$ in our numerical studies, which will be relaxed later.

All examples were computed for $k$ in the compact interval $[0,2]$ with control range ${ }^{28} j \in[0,0.25]$ except for the variant of the convex-concave production function. For the algorithm from Section 3.1 we have used the numerical time step $h=0.05$ and an initial grid with 39 nodes. The final adapted grid consisted of 130 nodes. The range of control values was discretized using 101 equidistributed values. For the algorithm from Section 3.2 we used the time step $h=0.5$, in order to generate the discrete time model $\Psi$ we used a highly accurate extrapolation method. For this algorithm the range of control values was discretized using 51 equidistributed values. The domain covered by the grid was chosen to be $(k, B) \in[0,2] \times[0,3]$. The initial grid was chosen with 1024 cells, while the final adapted grids consisted of about 100000 up to 500000 cells, depending on the example. For

\footnotetext{
${ }^{28}$ In all our experiments larger control ranges did not yield different results.
} 
this algorithm the figures below always show the set $E_{\Gamma}$ which approximates the creditworthiness curve $B^{*}(k)$. Recall that the width of this set gives an estimate for the spatial discretization error.

\subsection{Quadratic Adjustment Cost}

The first case we explore will assume a concave production function $a k^{\alpha}, 0<$ $\alpha<1$ and quadratic adjustment costs, $b j^{\beta}$. Such a model has often been used as a benchmark model in economics. As model parameters we specify $\alpha=0.5, \beta=2, b=0.5, a=0.29$ and $\theta=0.1$. This specifies the most simplest variant of a dynamic decision problem which has often been employed in economics and which can be shown to exhibit solely one positive steady state equilibrium $k^{*}$. The creditworthiness curve is simply given by the present value borrowing constraint, since we here assume a constant credit cost and a debt equation as shown in equ. (6).

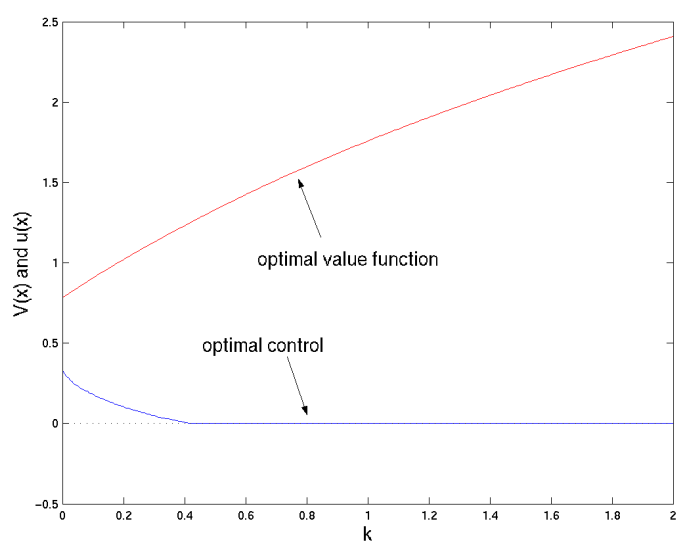

Figure 2: Optimal value function and feedback for example from Section 4.1

In this case we can use the optimal control algorithm of section 3.1. The creditworthiness curve is given by the optimal value function in figure 2 and the solution path of the dynamic decision problem, the investment decision, is given by the optimal control in figure 2. For the debt dynamics it holds that all initial levels of debt below the creditworthiness curve, can be steered bounded. 


\subsection{Convex-Concave Production Function}

Next we compute the creditworthiness curve for a model variant with a convex-concave production function as suggested by Skiba (1978). We disregard, as in Skiba, adjustment cost of investment but again presume a constant borrowing $\operatorname{cost} \theta=0.1$. The convex-concave production function is for our numerical purpose specified as a logistic function of $k$

$$
y=\frac{a_{0} \exp \left(a_{1} k\right)}{\exp \left(a_{1} k\right)+a_{2}}-\frac{a_{0}}{1+a_{2}}
$$

with $a_{0}=2500, a_{1}=0.0034, a_{2}=500$. This convex-concave production function takes the place of the production function $a k^{\alpha}$ in equ. (5), yet there is no adjustment cost term $j^{\beta} k^{-\gamma}$. The net income, $f(k, j)$, in equ. (5) is thus linear in the decision variable, $j$, and one would thus expect a bangbang solution. In our numerical solution we restrict the net income such that $f(k, j) \geq 0$. The results, using again the algorithm of Section 3.1, are shown in figure 3 .

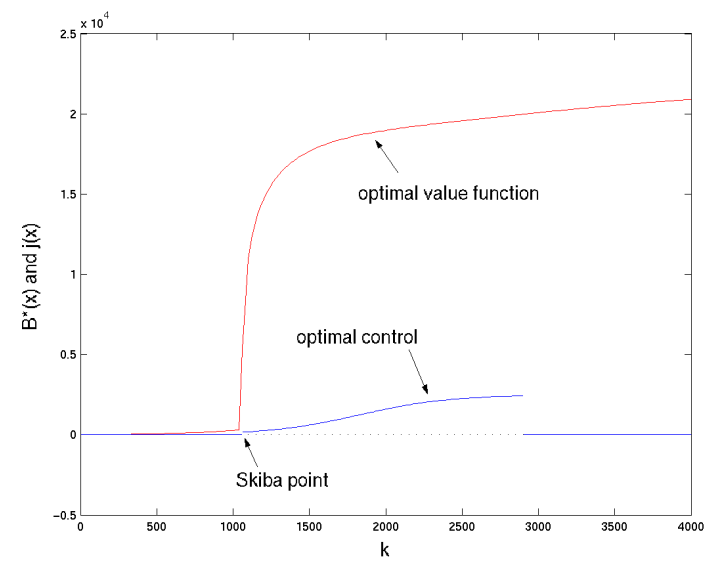

Figure 3: Optimal value function and feedback for example from Section 4.2

The optimal value function again demonstrates the creditworthiness curve and the optimal control, the dynamic decision problem. This variant of our model gives multiple steady states at 0 and 2847 which have been obtained by simulations, and a threshold, a Skiba point, at 1057 in the vicinity of which there is likely to be another, but non-optimal steady state.

Again any debt, $B_{0}$, below the creditworthiness curve can be steered bounded but capital stock with initial condition, $k_{0}$, to the left of the Skiba point will contract and to the right of the Skiba point will expand approaching 
the high steady state 2847. Thus the Skiba point is unstable and 0 and 2847 are attractors. As also clearly visible, at the Skiba point the control variable is discontinuous, it jumps. Note, however, that the jump of the control problem at the high steady state arises from the fact that, without adjustment cost, we have a decision problem linear in the decision variable.

\subsection{Adjustment Cost and Constant Credit Cost}

Next we consider a model variant with $a k^{\alpha}$ as production function in equ. (5) and the nonlinear adjustment cost term $j^{\beta} k^{-\gamma}$, but take $H(k, B)=\theta B$. We again can use the algorithm of Section 3.1 in order to solve the discounted infinite horizon problem (2)-(4). Figure 4 shows the corresponding optimal value function representing the creditworthiness curve (upper graph) and the related optimal control in feedback form (lower graph).

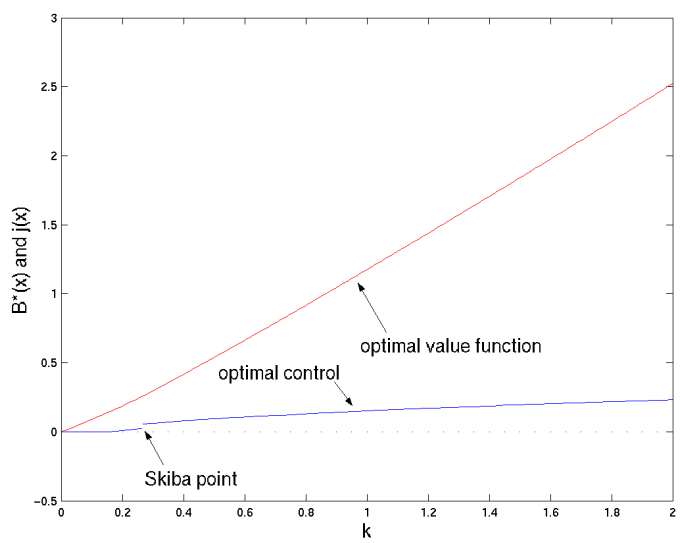

Figure 4: Optimal value function and feedback for example from Section 4.3

The threshold $k^{+}=0.267$ is clearly visible in the optimal control law, which is discontinuous at this point. For initial values $k(0)<k^{+}$the optimal trajectories tend to $k^{*}=0$, for initial values $k(0)>k^{+}$the optimal trajectories tend to the stable equilibrium $k^{* *}=0.996 .^{29}$

Figure 5 shows the optimal feedback control in a neighborhood of the threshold. The discontinuity in the control variable is clearly observable. Investment to the left of $k^{+}$is lower than $\sigma k$ and makes the capital stock

\footnotetext{
${ }^{29}$ Here again the non-negative equilibria are obtained by simulations. The equilibrium candidates can numerically be computed by using equ. (10).
} 
shrinking whereas investment to the right of $k^{+}$is larger than $\sigma k$ and increases the capital stock. At $k^{+}$investment jumps.

In addition, in this figure the adaptively distributed grid points are shown. As mentioned in Section 3.1, the grid is in particular refined around the threshold, the reason for this is the (barely visible) kink in the optimal value function at this point, resulting in a non-differentiable value function and hence in large local errors.

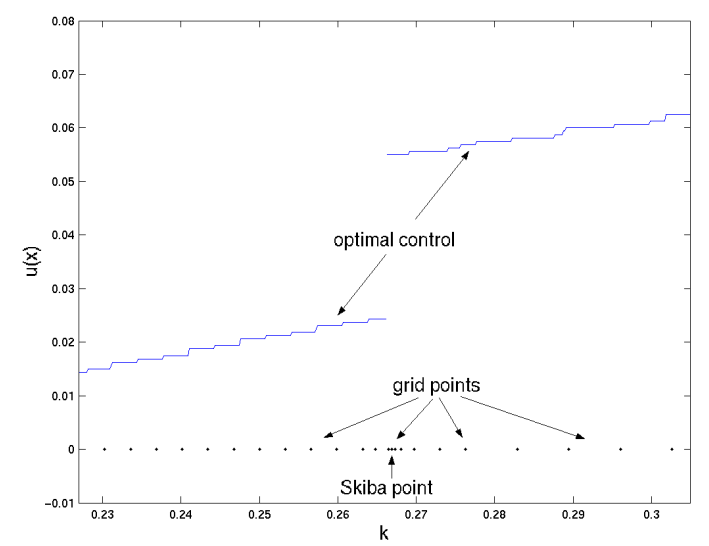

Figure 5: Optimal feedback law and distribution of grid points at threshold

\subsection{Adjustment Cost and Endogenous Credit Cost}

Next we consider a model variant which is the same as in Section 4.3 but with $H(k, B)$ from (1). For this more general model with

$$
H(k(t), B(t)))=\frac{\alpha_{1}}{\left(\alpha_{2}+\frac{N(t)}{k(t)}\right)^{\mu}} \theta B(t)
$$

it is not possible to transform the problem into a standard infinite horizon optimal control problem, hence we will use the algorithm for the computation of domains of attractions from Section 3.2 and undertake experiments for different shapes of the credit cost function.

In this formula we specify $\mu=2$. Taking into account that we want $\theta$ to be the risk-free interest rate, we obtain the condition $\alpha_{1} /\left(\alpha_{2}+1\right)^{2}=1$ and thus $\alpha_{1}=\left(\alpha_{2}+1\right)^{2}$. Note that for $\alpha_{2} \rightarrow \infty$ and $0 \leq B \leq k$ one obtains $H(k, B)=\theta B$, i.e., the model from the previous section. In order to compare 
these two models we use the formula $H(k, B)=\frac{\alpha_{1}}{\alpha_{2}^{2}} \theta B$ for $B>k .^{30}$

Figure 6 shows the respective creditworthiness curves $B^{*}$ under the condition $\sup _{t \geq 0} B(t)<\infty$ for $\alpha_{2}=100,10,1, \sqrt{2}-1$ (from top to bottom) and the corresponding $\alpha_{1}=\left(\alpha_{2}+1\right)^{2}$.

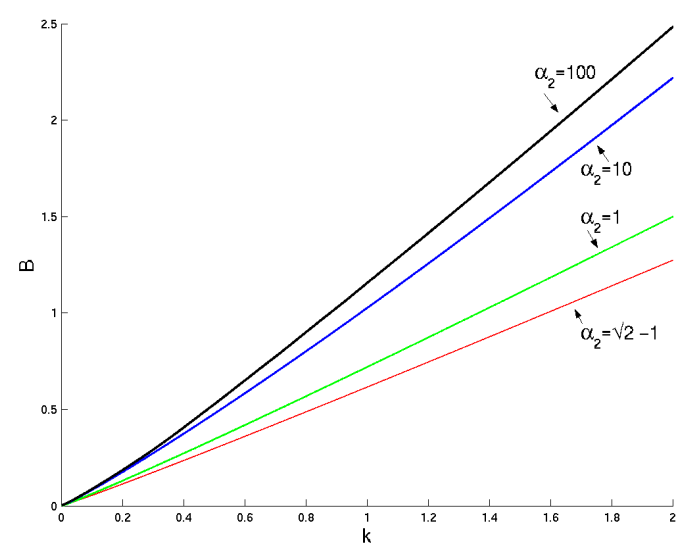

Figure 6: Creditworthiness curve $B^{*}$ for different $\alpha_{2}$

For $\alpha_{2}=100$ the trajectories on the curve $B^{*}$ show almost the same behavior as the optimal trajectories in the previous section: There exists a threshold (now at $k^{+}=0.32$ ) and two stable equilibria at $k^{*}=0$ and $k^{* *}=0.99{ }^{31}$ Further simulations have revealed that for decreasing values of $\alpha_{2} \leq 100$ the threshold value $k^{+}$increases (i.e., moves to the right) and the stable equilibrium $k^{* *}$ decreases (i.e., moves to the left), until they meet at about $\alpha_{2}=31$. For all smaller values of $\alpha_{2}$ there exists just one equilibrium at $k^{*}=0$ which is stable. The reason for this behavior lies in the fact that for decreasing $\alpha_{2}$ credit becomes more expensive, hence for small $\alpha_{2}$ it is no longer optimal to borrow large amounts and to increase the capital stock, instead it is optimal to shrink the capital stock and to reduce the stock of debt $B(t)$ to 0 . Thus, with small $\alpha_{2}$ and thus large borrowing cost it is for any initial capital stock optimal to shrink the capital stock.

From the figure 6 one can observe that, as our equ. (11) predicts, the creditworthiness curve moves down with a steeper slope of the credit cost function, equ. (1), which gives rise to a larger $V_{H}\left(k, B^{*}\right)$ in equ. (11).

\footnotetext{
${ }^{30}$ For small values of $\alpha_{2}$ it turns out that the creditworthiness curve satisfies $B^{*}(k)<k$, hence this change of the formula has no effect on $B^{*}$.

${ }^{31}$ Those are again obtained by simulations. The candidates for equilibria for the case of $H(k, B)$ can be computed by equ. (9).
} 


\begin{tabular}{cccc}
\hline$\alpha_{2}$ & 100 & 10 & $\sqrt{2}-1$ \\
\hline$V_{H}\left(k, B^{*}(k)\right)$ & 0.041 & 0.274 & 1.140 \\
$B^{*}(k)$ & 2.477 & 2.214 & 1.273 \\
$V(k)$ & 2.518 & 2.488 & 2.410
\end{tabular}

Table 1: Optimal values

In order to evaluate the extent wo which a value loss occurs, due to the finance premium of equ. (1), we compute for equ. (11) the component parts of $V(k)$ for different slopes of the credit cost function. Hereby the lower $\alpha_{2}$ represents a higher finance premium. As table 1 shows the numerical values obtained for the different $\alpha_{2}$ indicate that the value loss, $V_{H}(k, B)$, substantially increases, the creditworthiness, $B^{*}(k)$, substantially decrease and the value $V(k)$ slightly decreases with rising finance premium (falling $\left.\alpha_{2}\right)$.

\subsection{Adjustment Cost and Convex Credit Cost}

Next we specify the endogenous credit cost as $H(k, B)=\theta B^{\kappa}$. In this section we use the algorithm from Section 3.2 and repeat the computations from the previous section for the credit cost function $H(k, B)=\theta B^{\kappa} .{ }^{32}$ Figure 7 shows the respective curves for $\kappa=1,1.05,1.25,2$ (from top to bottom at the right boundary of the diagram).

For $\kappa=1$ this is exactly the optimal value function from Figure 4, while for increasing $\kappa$ the values of $B^{*}(k)$ increase for small $k$ and decrease for larger $k$. This is due to the fact that for increasing $\kappa$ and $B>1$ the credit cost increases whereas for increasing $\kappa$ and $B<1$ the credit cost decreases, hence it is becomes possible to borrow larger amounts with small capital stock. It should also be noted that in all cases we have $B^{*}(0)=0$, however, for larger $\kappa$ the creditworthiness curve becomes discontinuous at 0, i.e., $\lim _{k \rightarrow 0, k>0} B^{*}(k)>0$. Again, this is due to the fact that for larger $\kappa$ the credit cost is small for small $B$.

This behavior of the creditworthiness is also reflected in the thresholds. For $\kappa=1.05$ the qualitative behavior of the trajectories is just as in the case $\kappa=1$ : there exists a threshold $k^{+}>0$ where the control is discontinuous and there are two stable equilibria $k^{*}=0$ and $k^{* *}>k^{+}$. For increasing values of $\kappa$ the threshold $k^{+}$moves to the left until it hits 0 and vanishes;

\footnotetext{
${ }^{32}$ Note that this type of interest cost where the interest payment is convex in the agent's debt is frequently posited in the literature, see for example Bhandary, Haque and Turnovsky (1990) Again, equ. (9) can be used for computing equilibrium candidates.
} 


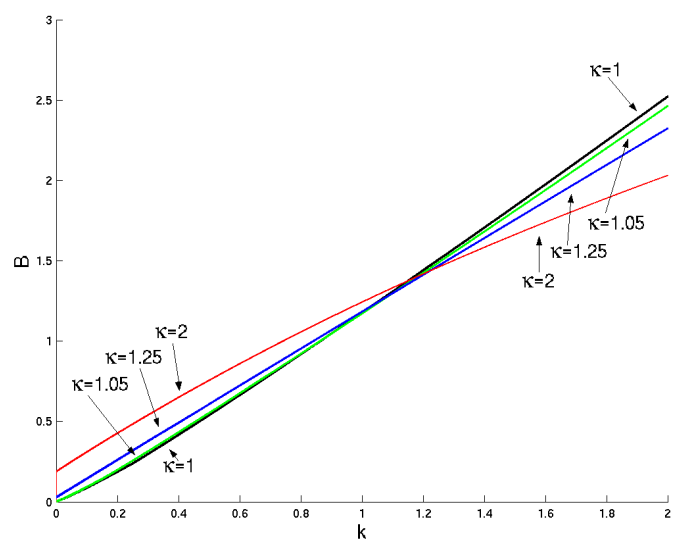

Figure 7: Creditworthiness curve $B^{*}$ for different $\kappa$

for $\kappa=1.25$ and $\kappa=2$ it has already vanished, implying that all trajectories with initial values on the $B^{*}(k)$ curve converge to a strictly positive stable equilibrium $k^{* *}$. Note that this behavior is just the opposite to what happens for $H(k, B)$ from (1) in Section 4.4 for decreasing $\alpha_{2}$, which is due to the fact that the credit cost for small $B$ behaves the opposite way. We can thus observe that for both type of credit cost functions the asset price and thus the creditworthiness is affected, yet for the convex credit cost the asset price decreases (relative to the a credit cost with risk-free rate) only for large capital stock and borrowing. This rather unexpected behavior of the convex credit cost function - increasing creditworthiness with small capital stock and borrowing - makes the first formulation of endogenous credit cost, through equ. (1), a more reasonable approach to pursue. On the other hand one might argue that small firms might need to be advantaged in order to allow them to enjoy a take-off. In this case a credit cost function of the type $H(B)=\theta B^{\kappa}$ appears to be more reasonable.

\subsection{Debt Ceilings}

Next we explore an extension of our model by introducing debt ceilings. For $H(k, B)$ from (1) with $\alpha_{2}=100$ and for $H(k, B)=\theta B^{\kappa}$ with $\kappa=2$ we now impose the restriction $B(t) / k(t) \leq c$ for some constant $c$. Again we use the algorithm from Section 3.2 where now in the definition of $D_{\Gamma}$ we only take into account those cell images which satisfy the restriction. Figure 8 shows the respective curves without restriction and for the restriction with $c=1.2$ and $c=0.6$ (from top to bottom). In addition, the restriction curves $B=c k$ 
are shown with dots for $c=1.2$ and $c=0.6$.

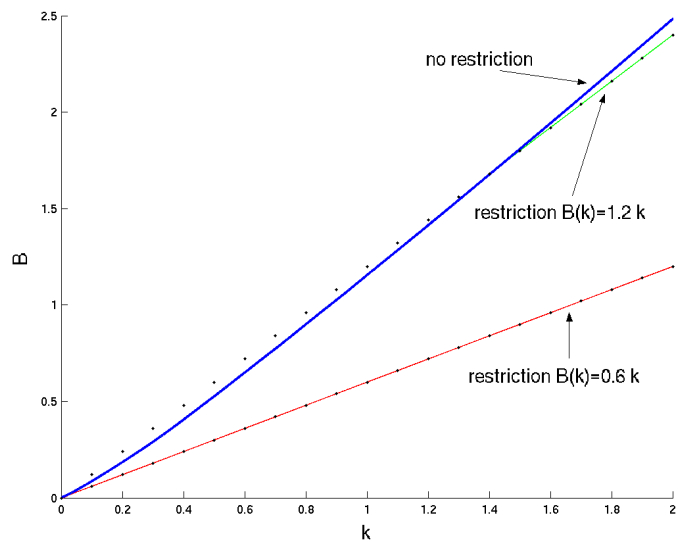

Figure 8: Creditworthiness curve $B^{*}$ for different ceilings, $H(k, B)$ from (1)

For $c=0.6$ the creditworthiness curve $B^{*}(k)$ coincides with the "restriction curve" $B(k)=c k$; in this case the curve $\left(k, B^{*}(k)\right)$ is no longer invariant for the dynamics, ${ }^{33}$ i.e., each trajectory $B(t)$ with $B(t) \leq B^{*}(k(t))$ leaves the curve $\left(k, B^{*}(k)\right)$ and reaches $B(t)=0$. For $c=1.2^{34}$ the curves $B^{*}(k)$ and $B=c k$ coincide only for $k \geq 1.46$. Here one observes the same equilibria $k^{*}$ and $k^{* *}$ and threshold $k^{+}$as for the sup-restriction (see Section 4.4), however, in addition to these here a new threshold appears at $k^{++}=1.54$. For initial values $\left(k, B^{*}(k)\right)$ with $k^{+}<k<k^{++}$the trajectory tends to the stable equilibrium $k^{* *}$, while for $k>k^{++}$the behavior is the same as for $c=0.6$, i.e., the corresponding trajectories leave the curve $B^{*}(k)$ and eventually $B(t)$ reaches zero. ${ }^{35}$

We have repeated these computations for $H(k, B)=\theta B^{\kappa}$ and $\kappa=2$. Figure 9 shows the respective curves for the restriction $\sup _{t>0} B(t)<\infty$ and for the ratio-restriction with $c=1.2$ and $c=0.6$ (from top to bottom). In addition, the restriction curves $B=c k$ are shown with dots for $c=1.2$ and $c=0.6$.

\footnotetext{
${ }^{33}$ As mentioned in Section 3.2 the boundary of the domain of attraction is invariant for the trajectories if no explicit restriction is active. In the case of a debt ceiling, however, this invariance property is no longer valid.

${ }^{34}$ This curve is difficult to see because it coincides with the curve without restriction for small $k$ and with the restriction curve $B=c k$ for large $k$.

${ }^{35}$ The simulation are halted at zero, but we would like to report if continued the $B(t)$ curve becomes negative and tends to $-\infty$.
} 


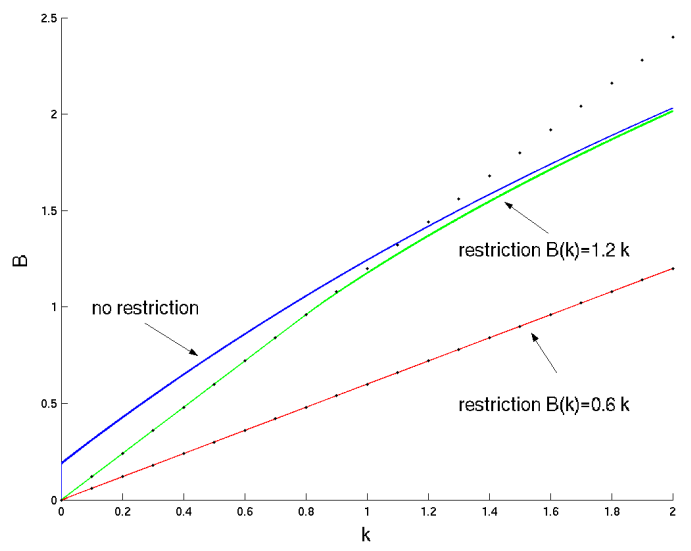

Figure 9: Creditworthiness curve $B^{*}$ for different ceilings, $H(k, B)=\theta B^{2}$

Again, without restriction the curve is discontinuous at $k=0$. Just as for $H(k, B)$ from $(1)$, for $c=0.6$ the creditworthiness curve $B^{*}(k)$ coincides with the "restriction curve" $B(k)=c k$ and the curve $\left(k, B^{*}(k)\right)$ is no longer invariant for the dynamics and for each trajectory with $B(t) \leq B^{*}(k(t))$ the second component $B(t)$ reaches 0 . For $c=1.2$ the curves $B^{*}(k)$ and $B(k)=c k$ coincide for $k \in\left[0, k^{* *}\right]$, where $k^{* *}=0.8$ is exactly the stable equilibrium for all trajectories starting on the curve $B^{*}(k)$.

\subsection{Consumption}

Note that in equ. (2)-(4) and the subsequent analytical study in sect. 2, we have treated consumption as exogenous and predicted that the exogenous constant consumption will move down the creditworthiness curve. Therefore, we next investigate -again for $H(k, B)$ from (1) with $\alpha_{2}=100$ and for $H(k, B)=\theta B^{\kappa}$ with $\kappa=2$ - the case when the agent's net income $f$ is reduced by a constant consumption $c(t) \equiv \eta$. In this case the creditworthiness curve $B^{*}$ may become negative. This means that there is an initial level of capital stock required - the level of capital stock where the creditworthiness curve becomes positive - that supports the consumption path $c(t)=\eta$. All levels of capital stock below this point do not support the consumption path $c(t)=\eta$. We have to specify the dynamics for $B(t)<0$ which we choose to be $\dot{B}(t)=\theta B(t)-f$. For this model we also choose the set $A$ as $A=$ $\{(k, B) \mid B \leq-1\}$ in order to allow the extension of the $B^{*}$-curve to $B<0$.

Note that for the linear model from Section 4.3 subtracting a constant $\eta$ from $f$ simply results in an optimal value function $V_{\eta}=V-\theta \eta$. Since for 
$\alpha_{2}=100$ the creditworthiness $B^{*}$ for $H(k, B)$ from $(1)$ is very close to the model from Section 4.3 we would expect much the same behavior. Figure 10 shows that this is exactly what happens here.

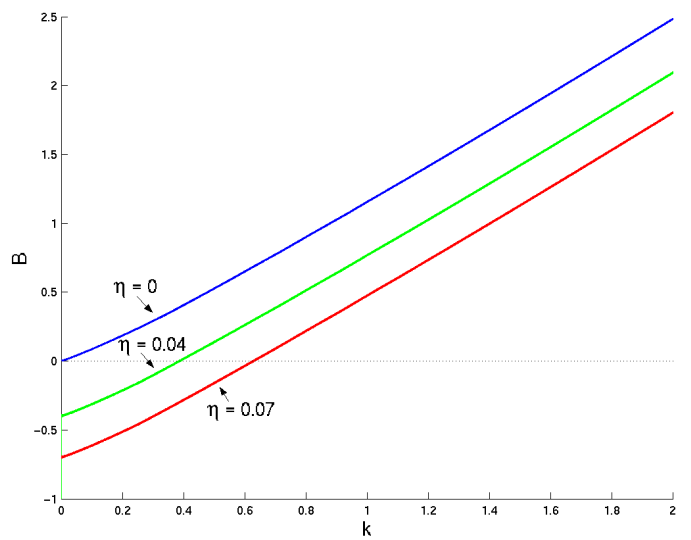

Figure 10: Creditworthiness curve $B^{*}$ for different $\eta, H(k, B)$ from $(1)$

The fact that the curves here are just shifted is also reflected in the stable equilibria and the threshold, which do not change their positions. In particular, the dynamical behavior does not depend on the consumption rate. $^{36}$

Again, we have repeated our computations with $H(k, B)=\theta B^{\kappa}$ and $\kappa=2$. Figure 11 shows the respective curves for $\eta=0,0.04,0.07$, again from top to bottom.

In this nonlinear model here with $\kappa=2$ the effect of $\eta$ is truly nonlinear, as it is easily seen from the figure, because the difference between the curves at the right boundary of the diagram is much smaller than on the left boundary. However, again the dynamical behavior does not change: just as for $\eta=0$, for both considered positive values of $\eta$ the resulting trajectories converge to a stable equilibrium $k^{* *}>0$ and no thresholds could be observed. The position of the equilibria $k^{* *}$ depends on $\eta$, more precisely $k^{* *}$ increases, i.e., moves to the right as $\eta$ increases.

As concerns the behavior of the creditworthiness curve for our two different credit cost functions we here too see a slight difference in the outcome for our convex credit cost function as compared to our function represented by equ. (1).

\footnotetext{
${ }^{36}$ Note that this is an obvious case where our separation theorem of section 2 is valid.
} 


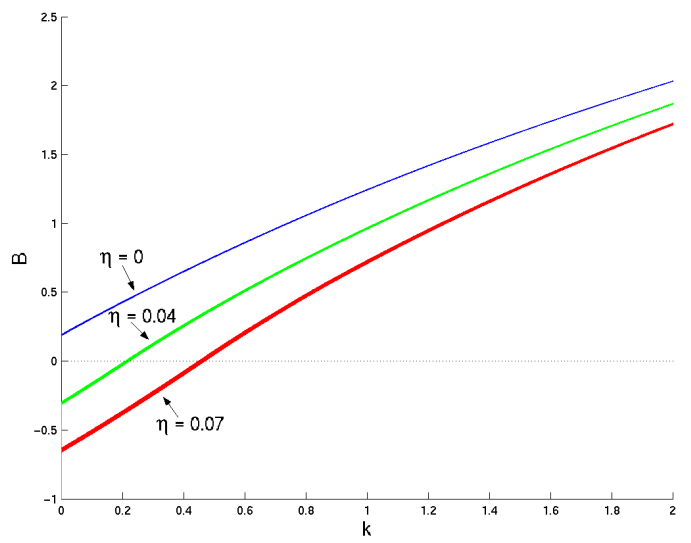

Figure 11: Creditworthiness curve $B^{*}$ for different $\eta, H(k, B)=\theta B^{2}$

\section{Conclusions}

We study a credit market model where the agents can borrow from credit market for investment and where the credit cost may be state dependent and the agents may face debt ceilings. The basis of the credit market model is a dynamic investment decision model with adjustment cost with imperfect capital markets due to asymmetric information and costly state verification. Borrowing cost becomes endogenously determined by endogenous collaterals of firms, given by a creditworthiness curve, or borrowing may be constrained by exogenous debt ceilings. The shape of creditworthiness curve is theoretically and numerically explored for a variety of model variants pertaining to the shape of the production function, the adjustment cost of capital and specifications of the imperfect capital markets.

Some of the model variants give rise to multiple steady state equilibria. Those model variants imply history dependence. The optimal path for capital and debt depend on initial conditions where the credit cost and constraints may interact. In the model variant with a unique positive steady state the initial debt is constrained in the sense that it must be below the creditworthiness curve which is in the standard model the present value borrowing constraint. In the model variants with multiple steady states the initial conditions for both debt and capital stock as well as possible debt ceilings - if imposed on the firm - matter for investment and thus for the path of the capital stock. Computing creditworthiness in the case of a unique steady state equilibrium is straight forward and may be even undertaken by employing the Hamiltonian equation but the computation of the creditworthiness curve 
with endogenous credit cost, debt ceiling and a given consumption path is more cumbersome.

In this paper we apply dynamic programming with flexible grid size that proves to be useful to compute creditworthiness, thresholds and the global dynamics. Using this method we can determine the present value borrowing constraint and thus the region in which the borrower remains creditworthy. We can detect thresholds, domains of attraction, jumps in the policy function and suboptimal equilibria. Finally we want to note that our model is nested in utility theory but can be studied separately. The model implies that neither the firm, in its dynamic investment decisions, nor the household, in its consumption decisions, face a period by period constraint. The net worth generated by the firms optimal investment decision feed finally into consumption but the firm is not constrained by each period's dividend payment. Consumption faces solely an intertemporal constraint whereby the sequence of consumption decisions can be based on any preference.

\section{References}

[1] Abel, A. and O. Blanchard (1986), "The Present Value of Profit and Cyclical Movements in Investment", Econometrica, vol. 54, no. 2: 249273

[2] Azariadis, C. and A. Drazen (1990), "Threshold Externalities in Economic Development", Quarterly Journal of Economics, vol. 105, no 2: 501-526.

[3] Bardi, M. and I. Capuzzo Dolcetta (1997), Optimal Control and Viscosity Solutions of Hamilton-Jacobi-Bellman equations, Birkhäuser, Boston.

[4] and Evidence", Carnegie-Rochester Conference Series on Policy 43: 163211.

[5] Benhabib, J. and S. Schmitt-Grohe and M. Uribe (1998), "Monetary Policy and Multiple Equilibria", Economic Research Reports, C.V. Starr Center for Applied Economics, New York University, RR \#98-02.

[6] Bernanke, B., Gertler, M. and S. Gilchrist (1999), "The Financial Accelerator in a Quantitative Business Cycle Framework", in J. Taylor and M. Woodford (eds), Handbook of Macroeconomics, Amsterdam, NorthHolland. 
[7] Beyn, W.-J., T. Pampel and W. Semmler (2001), "Dynamic Optimization and Skiba Sets in Economic Examples", Journal of Optimal Conrol Applications and Methods, 22: 251-280.

[8] Evans, G., S. Honkapohja and P. Romer (1997) "Growth Cycles", manuscript Stanford University.

[9] Bhandary, J. S., N. U. Haque and S. J. Turnovsky (1990), "Growth, External Debt, and Sovereign Risk in a Small Open Economy", IMF Staff Papers, vol. 37, no.2 International Monetary Fund.

[10] Blanchard O.J. (1983) "Debt and Current Account Deficit in Brazil" in P.A. Armella, R. Dornbush and M. Obstfield, eds., Financial Policies and the World Capital Market: The Problem of Latin American Countries, Chicago: University of Chicago Press, pp.187-197.

[11] Blanchard, O.J. and S. Fischer (1989), Lectures on Macroeconomics, Cambridge: MIT Press.

[12] Brock, W.A. and A.G. Malliaris (1996), Differential Equations Stability and Chaos in Dynamic Economics, Amsterdam: North-Holland.

[13] Brock, W. and D. Starret (1999), Nonconvexities in Ecological Management Problems, University of Wisconsin, mimeo.

[14] Capuzzo Dolcetta, I. (1983), On a Discrete Approximation of the Hamilton-Jacobi Equation of Dynamic Programming, Appl. Math. Optim., vol. 10, pp. 367-377.

[15] Carlstrom, C.T. and T.S. Fuerst (1997), Agency Cost, Net Worth and Business Fluctuations: A Compatible General Equilibrium Analysis, The American Economic Review, December, pp: 893-910

[16] Cooley, T. and V. Quadrini (1998) "Monetary Policy and the Financial Decisions of Firms", University of Rochester, mimeo

[17] Deissenberg, C., G. Feichtinger, W. Semmler and F. Wirl (2003), "History Dependence and Global Dynamics in Models with Multiple Equilibria", forthcoming.

[18] Eaton, J. and R. Fernandez (1995), "Sovereign Debt", National Bureau of Economic Research working paper series, no 5131. 
[19] Falcone, M. (1987), A Numerical Approach to the Infinite Horizon Problem of Deterministic Control Theory, Appl. Math. Optim., vol. 15, pp. 113. Corrigenda (1991), ibid., vol. 23, 213-214.

[20] Greiner, A. and W. Semmler (2002), "Monetary Policy, and Hysteresis Effects on the Labor Market", in R. Neck (ed.), Modelling and Control Economic Systems, Elsevier Sciences, Ltd., Oxford.

[21] Grüne, L. (1997), "An Adaptive Grid Scheme for the Discrete HamiltonJacobi-Bellman Equation", Numerische Mathematik vol. 75, pp. 9-337.

[22] Grüne, L, M. Metscher and M. Ohlberger (1999), On Numerical Algorithm and Interactive Visualization for Optimal Control Problems, Comput. Vis. Sci., vol. 1, pp. 221-229.

[23] Grüne, L. (2001), Subdivision Techniques for the Computation of Domains of Attraction and reachable Sets, Proc. of NOLCOS 2001, St. Petersburg, Russia, pp. 762-767.

[24] Grüne, L. (2002), Asymptotic Behavior of Dynamical and Control Systems under Perturbation and Discretization, Lecture Notes in Mathematics 1783, Berlin: Springer Verlag.

[25] Hartl, R., P.M. Kort, G. Feichtinger and F. Wirl (2003), "Multiple Equilibria and Thresholds due to Relative Investment Costs: Non-Concave Concave, Focus-Node, Continuous - Dicontinuous", forthcoming in Journal of Optimization Theory and Applications.

[26] Kiyotaki, N. and J. Moore (1997) Credit Cycles, Journal of Political Economy, vol 105, April:211-248.

[27] Krieger, S. (1999), "Bankruptcy Costs, Financial Constraints and the Business Cycle", Yale University, mimeo

[28] Krugman, P. (1991), History versus Trade, Quarterly Journal of Economics, vol. :651-667.

[29] Miller, M. and J. Stiglitz (1999), Bankruptcy Protection against Macroeconomic Shocks, mimeo, The World Bank.

[30] Mortensen, D. T. (1989), The Persistence and Indeterminacy of Unemployment in Search Equilibria, Scadinavian Journal of Economics, 91, (2):347-370. 
[31] Orphanides, A. and Zervos (1998), Myopia and Addictive Behavior, Economic Journal, vol. 108:75-91

[32] Santos, M.S. (1999), Non-Existence of Continuous Markov Equilibria in Competitive Market Economies, University of Minnesota, mimeo.

[33] Semmler, W. and M. Sieveking (1996), "Computing Creditworthiness and Sustainable Debt", Dept. of Mathematics, University of Frankfurt, paper presented at the Conference on "Computing in Economics and Finance", University of Geneva, June, 1996.

[34] Semmler, W. and M. Sieveking (1999), Using Vector Field Analysis for Studying Debt Dynamics, paper presented at the North American Winter Meeting of the Econometric Society, Chicago, 1998, Bielefeld University, Discussion paper no. 379.

[35] Semmler, W. and M. Sieveking (2000), Critical Debt and Debt Dynamics, Journal of Economic Dynamics and Control, vol 24:1121-44.

[36] Sieveking, M. and W. Semmler (1999), Credibility, Dept. of Economics, University of Bielefeld, mimeo

[37] Sieveking, M, and W. Semmler (1998), The Optimal Consumption of Value, Dept. of Economics, University of Bielefeld, mimeo.

[38] Sieveking, M. and W. Semmler (1997), "The Present Value of Resources with Large Discount Rates", Applied Mathematics and Optimization: An International Journal, 35:283-309.

[39] Skiba, A.K. (1978), "Optimal Growth with Convex-Concave Production Function", Econometrica, 46:527-539

[40] Townsend, R. (197), "Optimal Contracts and Competitive Markets with Costly State Verification", Journal of Economic Theory, 21: 265-293.

[41] Turnovsky, S. J. (1995), Methods of Macroeconomic Dynamics, Cambridge: MIT Press.

[42] Wirl, A. and G. Feichtinger (2000), History Dependence Due to Unstable Steady States on Concave Intertemporal Optimization, University of Technology, Vienna, mimeo 